\title{
The Power of Test of Jennrich Statistic with Robust Methods in Testing the Equality of Correlation Matrices
}

\author{
Bahtiar Jamili Zaini*, Shamshuritawati Md Sharif \\ School of Quantitative Sciences, Universiti Utara Malaysia, 06010 UUM Sintok, Kedah, Malaysia
}

Received August 27, 2021; Revised December 10, 2021; Accepted December 21, 2021

\section{Cite this paper in the following Citation Styles}

(a): [1] Bahtiar Jamili Zaini, Shamshuritawati Md Sharif, "Power of Test of Jennrich Statistics with Robust Methods in Testing the Equality of Correlation Matrices," Mathematics and Statistics, Vol. 10, No. 1, pp. 215 - 221, 2022. DOI: 10.13189/ms.2022.100120.

(b): Bahtiar Jamili Zaini, Shamshuritawati Md Sharif (2022). Power of Test of Jennrich Statistics with Robust Methods in Testing the Equality of Correlation Matrices. Mathematics and Statistics, 10(1), 215 - 221. DOI: 10.13189/ms.2022.100120.

Copyright $\odot 2022$ by authors, all rights reserved. Authors agree that this article remains permanently open access under the terms of the Creative Commons Attribution License 4.0 International License

\begin{abstract}
Jennrich statistic is a method that can be used to test the equality of 2 or more independent correlation matrices. However, Jennrich statistic begins to be problematic when there is presence of outliers that could lead to invalid results. When exiting outliers in data, Jennrich statistic implications will affect Type I errors and will reduce the power of test. To overcome the presence of outliers, this study suggests the use of robust methods as an alternative method and therefore, it will integrate the estimator into Jennrich statistic. Thus, it can improve the testing performance of correlation matrix hypotheses in relation to outlier problems. Therefore, this study proposes 3 statistical tests, namely $J$ s-statistic, $J m$-statistic, and Jmad-statistic that can be used to test the equation of 2 or more correlation matrices. The performance of the proposed method is assessed using the power of test. The results show that $\mathrm{Jm}$-statistic and Jmad-statistic can overcome outlier problems into Jennrich statistic in testing the correlation matrix hypothesis. Jmad-statistic is also superior in testing the correlation matrix hypothesis for different sample sizes, especially those involving $10 \%$ outliers.
\end{abstract}

Keywords Correlation, Jennrich Statistic, Robust Correlation Methods, Outlier

\section{Introduction}

In statistical analysis, testing several correlation matrices play a vital role to measure the linear relationship between different set of groups. This estimation of parameter correlation matrices from samples serves a particularly important role in high-dimensional data analysis, including the principal component analysis [1], [2], discriminant analysis [3], clustering analysis [4], and regression analysis [5], [6]. The importance of equality of several correlation matrices has been shown in many areas such as economics, financial market, medicine, and social science [7].

Generally, testing the equality of correlation matrices have been proposed and studied by several researchers. The early development in testing the correlation matrices methods can be found in many literature, for example Box (1949) [8], Kullback (1967) [9], Aitkin et al. (1968) [10], Aitkin (1969) [11], and Jennrich (1970) [12]. Jennrich statistic is a method for testing the equality of 2 or more independent samples correlation matrices [12]-[14]. This test is constructed based on the likelihood ratio test proposed by Kullback (1967) [9] and it is applied to a sequence of independent samples of correlation matrix based on samples from multivariate normal distribution [15]. This statistic also has much better computational and distributional properties [16].

Jennrich statistic, on the other hand, relies heavily on Pearson correlations in calculating test statistical values. Although Pearson correlation is the most frequently used method to determine the strength of relationships between variables because of its superior performance, especially when data follow a normal distribution with linear relationships, these coefficients have problems when data sets contain outlines [13], [17]. Statistical results will be 
poor if outliers are present. The presence of these outliers resulted in damaging effects for statistical analysis with increase of variance error and decrease of statistical test power [17]-[19]. If there are outliers in the data, the regression analysis will result in a divergence from normality, making the forecast value invalid [20]. As a result, Jennrich statistic is very sensitive to outliers, which can lead to erroneous conclusions. Jennrich statistic implications will affect type I errors and reduce the power of test. A robust method can be used as an alternative to reducing the influence of outliers. By using the robust estimator, classical estimator can be replaced to reduce the influence of outliers [21]. Studies initiated by Huber related to robust methods have been successfully applied to statistics especially in multivariate analysis [22]. More importantly, this method performs well with data drawn from non-normal distribution and could give approximately the same results as the classical method even when there is absence or presence of small proportion of outliers in data [23].

The hypothesis of 2 correlation matrices will be tested using traditional Jennrich statistics. However, this statistic is highly sensitive to the outliers. Therefore, this study will integrate robust correlation to overcome outlier problems into Jennrich statistic in testing correlation matrix hypothesis. In addition, this study also focuses on developing robust Jennrich statistic. Then, the performance of Jennrich statistic by integrating the robust correlation coefficient in the presence of outliers will be evaluated using the power of test with simulation study.

\section{Methodology}

This study proposes 3 new integration methods of robust correlation into Jennrich statistic to overcome outlier problems. These 3 robust measures of correlation are Spearman's rank correlation coefficient, median correlation coefficient, and median absolute deviation correlation coefficient. The aim of this research is to integrate robust correlation into Jennrich statistic that can improve the performance of hypothesis testing of correlation matrices in relations to outlier problems. Therefore, this study focuses on developing robust Jennrich statistic. The performance of the developed robust Jennrich statistic is evaluated using power of test.

\subsection{Jennrich Statistic}

Jennrich statistic is used to test the equality of 2 independent samples of correlation matrices. Consider the null hypothesis $H_{0}: P_{1}=P_{2}$ versus alternative hypothesis $H_{1}: P_{1} \neq P_{2}$ where $P_{1}$ and $P_{2}$ are the correlation matrices. To test this hypothesis, Jennrich proposes to use this following statistic [12],

$$
J=\frac{1}{2} \operatorname{tr}\left(Z^{2}\right)-\left(Z_{d g}\right)^{t} W^{-1} Z_{d g}
$$

where

$$
Z=\bar{R}^{-1}\left(R_{1}-R_{2}\right) \sqrt{\frac{n_{1} n_{2}}{n_{1}+n_{2}}}
$$

$R_{1}$ and $R_{2}$ represent sample correlation matrices with $n_{1}$ observations and $n_{2}$ observations, respectively

$$
\bar{R}=\frac{n_{1} R_{1}+n_{2} R_{2}}{n_{1}+n_{2}} \text { is the pooled correlation matrix }
$$

$W=I_{p}+R_{p} * R_{p}^{-1}$ is Hadamard product of 2 matrices (4)

$I_{p}$ is the identity matrix of size $(p \times p)$

Jennrich (1970) shows that the statistical test, $J$ is asymptotically $\chi^{2}$ distributed with degree of freedom $k=$ $p(p-1) / 2$ where $p$ is dimension of the correlation matrix [12]. Therefore, the null hypothesis $H_{0}: P_{1}=P_{2}$ is rejected at level of significance, $\alpha$ if $J$ exceeds $\chi_{\alpha, k}^{2}$, the $(1-\alpha)-$ th quantile of $\chi^{2}$ distribution.

\subsection{Correlation Method}

The Pearson correlation coefficients are commonly employed in parametric tests to assess the strength of a two-factor relationship between variables. For the nonparametric test, the Spearman rank correlation coefficient and the Kendall Tau correlation coefficient were employed to assess the strength of the relationship between the 2 variables. A robust correlation methodology can be used to compute the correlation between 2 variables as an alternate way, especially involving outlier data. Under deviation from the assumed distribution model, a strong correlation method can ensure that the statistical stability of the conclusion is high [24], [25]. Zaini and Sharif (2020) [26] compared to the performance of 5 types of correlation, namely Pearson correlation, Spearman correlation, Kendall Tau correlation, median correlation, and median absolute deviation correlation. The findings demonstrated that the correlation of the median absolute deviation has a strong relationship compared to other methods when there are large outliers in data. Therefore, based on the findings by Zaini and Sharif (2020) [26], this study will use some of these correlations to integrate into Jennrich statistic.

\subsection{Proposed Method}

Generally, we embed 3 correlation methods in Jennrich statistic as follows:

1. Develop Spearman Jennrich Statistic by embedding the Spearman correlation in Jennrich statistic, namely Js-statistic:

$$
J_{S}=\frac{1}{2} \operatorname{tr}\left(Z s^{2}\right)-\left(Z s_{d g}\right)^{t} W^{-1} Z s_{d g}
$$

where

$$
Z s=R s_{p}^{-1}\left(R s_{1}-R s_{2}\right) \sqrt{\frac{n_{1} n_{2}}{n_{1}+n_{2}}}
$$

$R s_{1}$ and $R s_{2}$ represent sample correlation matrices with $n_{1}$ observations and $n_{2}$ observations, respectively based on 
Spearman correlation.

2. Develop median Jennrich Statistic by embedding median correlation method in Jennrich statistic, namely $\mathrm{Jm}$-statistic:

$$
J m=\frac{1}{2} \operatorname{tr}\left(Z m^{2}\right)-\left(Z m_{d g}\right)^{\mathrm{t}} W^{-1} Z m_{d g}
$$

where

$$
Z m=R m_{p}^{-1}\left(R m_{1}-R m_{2}\right) \sqrt{\frac{n_{1} n_{2}}{n_{1}+n_{2}}}
$$

$R m_{1}$ and $R m_{2}$ represent sample correlation matrices with $n_{1}$ observations and $n_{2}$ observations, respectively based on median correlation.

3. Develop median absolute deviation Jennrich Statistic by embedding median absolute deviation correlation method in Jennrich statistic, namely Jmad-statistic:

$$
J m a d=\frac{1}{2} \operatorname{tr}\left(Z_{m a d}^{2}\right)-\left(Z_{m a d}\right)^{\mathrm{t}} W^{-1} Z_{m a d}
$$

where

$$
Z m a d=\operatorname{Rmad}_{p}^{-1}\left(\operatorname{Rmad}_{1}-\operatorname{Rmad}_{2}\right) \sqrt{\frac{n_{1} n_{2}}{n_{1}+n_{2}}}
$$

$\mathrm{Rmad}_{1}$ and $\mathrm{Rmad}_{2}$ represent sample correlation matrices with $n_{1}$ observations and $n_{2}$ observations, respectively based on median absolute deviation correlation.

\subsection{Simulation Data}

We used simulation data to obtain $p$-value of the Jennrich statistic and proposed methods. We used simulation study by using $\mathrm{R}$ programming version 3.4.2. The simulation study is executed based on 10,000 replications to obtain an accuracy of a power approximation. For that purpose, we;

1. generate $2 n$ random data from $p$-variate normal distribution with a mean vector of $\mathbf{0}$ and covariance matrix, $\boldsymbol{\Sigma}=1$,

2. calculate the statistical test value of proposed methods,

3. replicate the simulation in step (1) and (2) for 10,000 times,

4. identify the critical value by determining the cut of value of $95 \%$ percentiles of proposed methods,

5. generate another $n$ random data from $p$-variate normal distribution with mean vector $\mathbf{0}$ and covariance matrix, $\boldsymbol{\Sigma}=0.1,0.2,0.3, \ldots$, until 0.9,

6. calculate the statistical test value of propose method,

7. replicate the simulation in steps (5) and (6) for 10,000, and

8. calculate the power of test.

In each data set, we consider different number of variables, $p$ with $p=2,5,10,20,30$, and 50 as well as different size of samples with $n=20,30,50$, and 100. For all pairs of variables and sample sizes, this study uses the same correlation shift, $r$ of $r=0,0.1,0.2,0.3 \ldots 0.9$.

\subsection{Power of Test}

The procedure is continued by comparing the power of test between the classical and the proposed methods. The concept of power is used to make a comparison between different statistical testing procedures, where the most powerful test will have a higher number of rejections of the null hypothesis [27]. Generally, the power of test is known as $1-\beta$, where $\beta$ is the Type II error probability. This power of test indicates the probability of rejecting a null hypothesis when it's false, or the probability of not making a type II error. Thus, the power of test is the ability of a test to correctly reject the null hypothesis. Yue et al. (2002) [28] estimated the power of test as:

$$
\text { Power of Test }=\frac{R_{n}}{B},
$$

where

$R_{n}$ is the number of experiments that fall in the rejection region, and

$B$ is the total number of experiments

\section{Results and Discussion}

\subsection{Performance Evaluation Based on Power of Test}

Table 1 displays the power of test for comparing correlation using classical Jennrich statistic with proposed method; $\mathrm{Js}, \mathrm{Jm}$, and $\mathrm{Jmad}$-statistic, different of sample sizes, $(n=10,20,30,50$, and 100), and different level of correlation, $(r=0.1,0.2 \ldots 0.9)$. 
Table 1. Power of Test for Different Methods and Different Correlation Shifts

\begin{tabular}{|c|c|c|c|c|c|c|c|c|c|c|}
\hline Method & $\mathbf{n}$ & 0.1 & 0.2 & 0.3 & 0.4 & 0.5 & 0.6 & 0.7 & 0.8 & 0.9 \\
\hline \multirow{5}{*}{$\begin{array}{l}\text { Jennrich } \\
\text { Statistic }\end{array}$} & 10 & 0.0076 & 0.0134 & 0.0298 & 0.0565 & 0.1034 & 0.176 & 0.286 & 0.5198 & 0.7883 \\
\hline & 20 & 0.0111 & 0.025 & 0.071 & 0.1618 & 0.3154 & 0.5165 & 0.7721 & 0.9517 & 0.9987 \\
\hline & 30 & 0.0115 & 0.0492 & 0.114 & 0.282 & 0.5252 & 0.7983 & 0.9536 & 0.9969 & 1 \\
\hline & 50 & 0.0188 & 0.0844 & 0.2586 & 0.555 & 0.8394 & 0.9761 & 0.9986 & 1 & 1 \\
\hline & 100 & 0.0392 & 0.2242 & 0.609 & 0.9155 & 0.9962 & 1 & 1 & 1 & 1 \\
\hline \multirow{5}{*}{$\begin{array}{l}\text { Spearman } \\
\text { Jennrich } \\
\text { Statistic, Js }\end{array}$} & 10 & 0.0113 & 0.0172 & 0.028 & 0.0523 & 0.0891 & 0.158 & 0.2689 & 0.4038 & 0.6496 \\
\hline & 20 & 0.0129 & 0.0311 & 0.0726 & 0.1448 & 0.2603 & 0.4579 & 0.6997 & 0.8841 & 0.9861 \\
\hline & 30 & 0.0171 & 0.0489 & 0.1206 & 0.2631 & 0.4626 & 0.7166 & 0.9046 & 0.9839 & 0.9998 \\
\hline & 50 & 0.0244 & 0.088 & 0.2374 & 0.5048 & 0.7635 & 0.9456 & 0.9926 & 0.9999 & 1 \\
\hline & 100 & 0.0386 & 0.2045 & 0.5479 & 0.8546 & 0.9862 & 0.9993 & 1 & 1 & 1 \\
\hline \multirow{5}{*}{$\begin{array}{c}\text { Median } \\
\text { Jennrich } \\
\text { Statistic, Jm }\end{array}$} & 10 & 0.0356 & 0.0388 & 0.0452 & 0.0524 & 0.0697 & 0.0961 & 0.1291 & 0.2006 & 0.3333 \\
\hline & 20 & 0.0342 & 0.0453 & 0.0637 & 0.0953 & 0.1294 & 0.1816 & 0.2898 & 0.4303 & 0.6857 \\
\hline & 30 & 0.0352 & 0.0524 & 0.0742 & 0.1219 & 0.1949 & 0.3104 & 0.4662 & 0.6703 & 0.89 \\
\hline & 50 & 0.0365 & 0.0709 & 0.1194 & 0.2117 & 0.3523 & 0.5288 & 0.7218 & 0.8934 & 0.9901 \\
\hline & 100 & 0.0509 & 0.11 & 0.239 & 0.4425 & 0.6419 & 0.8478 & 0.9648 & 0.9982 & 1 \\
\hline \multirow{5}{*}{$\begin{array}{l}\text { Median } \\
\text { Absolute } \\
\text { Deviation } \\
\text { Jennrich } \\
\text { Statistic, } \\
\text { Jmad }\end{array}$} & 10 & 0.0389 & 0.0353 & 0.0456 & 0.0507 & 0.0713 & 0.0833 & 0.1295 & 0.17 & 0.2797 \\
\hline & 20 & 0.0357 & 0.0483 & 0.0564 & 0.0838 & 0.1274 & 0.1966 & 0.2843 & 0.3985 & 0.6457 \\
\hline & 30 & 0.041 & 0.054 & 0.0789 & 0.1286 & 0.1925 & 0.3004 & 0.4513 & 0.6464 & 0.8933 \\
\hline & 50 & 0.0434 & 0.0689 & 0.1219 & 0.204 & 0.3397 & 0.527 & 0.7117 & 0.8943 & 0.9898 \\
\hline & 100 & 0.0518 & 0.1021 & 0.2321 & 0.4048 & 0.6526 & 0.8474 & 0.9605 & 0.998 & 1 \\
\hline
\end{tabular}

Based on Table 1, we found that,

i. for each method, the power value will increase if the sample size increases and the correlation shift also increases.

ii. the power value of classical Jennrich statistic is always greater than other statistics when the correlation is 0.4 and above for different sample size.

iii. the power value of Jmad-statistics is better compared to others when the correlation is low $(r=0.1)$ for different sample size.

iv. for small sample size, $n=10$, classical Jennrich statistics becomes powerful when the correlation is 0.8 . Meanwhile $J s$-statistic become powerful when $r=$ 0.9 . For $J m$-statistic and Jmad-statistic, both statistics are not powerful for all correlation shifts.

v. for small sample size $n=20$, classical Jennrich statistics become powerful when the correlation is 0.6. Meanwhile Js-statistic become powerful when $r=0.7$, $\mathrm{Jm}$-statistic and Jmad-statistic become power when $r$ $=0.9$.

vi. for sample size $n=30$, classical Jennrich statistics become powerful when the correlation is 0.5 , Js-statistic become powerful when $r=0.6$, $J m$-statistic become power when $r=0.8$ and Jmad-statistic become powerful when $r=0.8$.

vii. for large sample size $n=50$, classical Jennrich statistics and $J s$-statistic become powerful when the correlation is 0.4. Meanwhile $\mathrm{Jm}$-statistic and Jmad-statistic become powerful when $r=0.6$. viii. for large sample size $n=100$, classical Jennrich statistics and Js-statistic become powerful when the correlation is 0.3 , meanwhile $J m$-statistic and $J$ Jmad-statistic become powerful when $r=0.5$.

\subsection{Comparison Power of Test for Jennrich Statistics Using Different Percentage of Outlier}

Previously, we compared classical Jennich statistic with the proposed method; Js, Jm, and Jmad-statistic without the presence of outliers. Next, 2 data sets are formed where the first data set does not contain an outlier, while the second data set is formed by entering different percentages stating $10 \%, 20 \%, 30 \%, 40 \%$, and $50 \%$ of sample sizes. Then, we calculated the power of test of the hypothesis test to test the equality of 2 independent correlation matrices. The outcome of the power of test for Jennrich statistic, Js, $\mathrm{Jm}$, and Jmad-statistic with different percentage of outlier with $10 \%, 20 \%, 30 \%, 40 \%$, and $50 \%$ are presented in Table 2 until Table 6.

Based on Table 2, for a small sample size ( $n=10)$, when outlier data are in the dataset, the power of test for Jennrich statistic increased from 0.7396 when there is only $10 \%$ outlier to 0.9736 when there are $50 \%$ outliers in the data. This means that hypothesis testing using Jennrich statistic is very sensitive to the presence of outliers. However, $\mathrm{Jm}$-statistic and Jmad-statistic do not show high values of power of test for detecting a false null hypothesis when 
there is presence of outlier. Jm-statistic only show high values of power of test when there are $50 \%$ data outliers. Jmad-statistic, on the other hand, do not show high power of test values even with the presence of data outliers. These results show that Jmad-statistic are not affected by up to $50 \%$ outliers when performing hypothesis tests between 2 correlations, while $\mathrm{Jm}$-statistic are not affected by up to $40 \%$ outliers when performing hypothesis tests between 2 correlations.

Next, we continued to look at the power of test when we increase the number of sample size in the dataset. For sample size $n=20$, the power test value for Jennich statistics is still high although with the presence of only $10 \%$ outliers which is 0.9996 . Meanwhile Js-statistic showed a high value of power of test (0.5286) at the presence of 30\% outliers. Jm-statistics and Jmad-statistics also showed high power of test values when there is presence of $30 \%$ outliers of 0.684 and 0.5286 , respectively. This indicates that when the sample size is increased, all test statistics in detecting a false null hypothesis begin to demonstrate effects with the presence of $20 \%$ outliers.

Table 2. Power of test for different statistics with outliers for $n=10$

\begin{tabular}{|c|c|c|c|c|c|}
\hline & \multicolumn{5}{|c|}{$n=\mathbf{1 0}$} \\
\hline outlier & $\mathbf{1 0 \%}$ & $\mathbf{2 0 \%}$ & $\mathbf{3 0 \%}$ & $\mathbf{4 0 \%}$ & $\mathbf{5 0 \%}$ \\
\hline $\begin{array}{c}\text { Jennrich } \\
\text { statistic }\end{array}$ & 0.7396 & 0.8961 & 0.9428 & 0.9692 & 0.9736 \\
\hline JS-statistic & & & 0.663 & 0.7536 & 0.8529 \\
\hline Jm-statistic & 0.0004 & 0.0212 & 0.1491 & 0.3772 & 0.5913 \\
\hline Jmad-statistic & 0 & 0.0053 & 0.0854 & 0.2993 & 0.4172 \\
\hline
\end{tabular}

Table 3. Power of test for different statistics with outliers for $n=20$

\begin{tabular}{|c|c|c|c|c|c|}
\hline & \multicolumn{5}{|c|}{$n=\mathbf{2 0}$} \\
\hline outlier & $\mathbf{1 0 \%}$ & $\mathbf{2 0 \%}$ & $\mathbf{3 0 \%}$ & $\mathbf{4 0 \%}$ & $\mathbf{5 0 \%}$ \\
\hline $\begin{array}{c}\text { Jennrich } \\
\text { statistic }\end{array}$ & 0.9996 & 0.9999 & 1 & 1 & 1 \\
\hline Js-statistic & 0.4644 & 0.0486 & 0.5286 & 0.8272 & 0.8908 \\
\hline Jm-statistic & 0.0684 & 0.308 & 0.684 & 0.8779 & 0.9567 \\
\hline Jmad-statistic & 0.0022 & 0.0486 & 0.5286 & 0.8272 & 0.8908 \\
\hline
\end{tabular}

Table 4. Power of test for different statistics with outliers for $n=30$

\begin{tabular}{|c|c|c|c|c|c|}
\hline & \multicolumn{5}{|c|}{$n=30$} \\
\hline outlier & $\mathbf{1 0 \%}$ & $\mathbf{2 0 \%}$ & $\mathbf{3 0 \%}$ & $\mathbf{4 0 \%}$ & $\mathbf{5 0 \%}$ \\
\hline $\begin{array}{c}\text { Jennrich } \\
\text { statistic }\end{array}$ & 1 & 1 & 1 & 1 & 1 \\
\hline Js-statistic & 0.923 & 0.997 & 0.9999 & 1 & 1 \\
\hline Jm-statistic & 0.1406 & 0.5388 & 0.9093 & 0.9862 & 0.9973 \\
\hline Jmad-statistic & 0.0121 & 0.2015 & 0.8699 & 0.9839 & 0.9925 \\
\hline
\end{tabular}

Next, we continued increasing the sample size to $n=30$. As in Table 4, the power of test value for Jennrich statistic still gives high values with the presence of only $10 \%$ outliers. Js-statistic also showed high value of power of test at the presence of $10 \%$ outliers. Jm-statistics showed high value of power of test when there is a presence of $20 \%$ outliers and Jmad-statistics maintained a high power of test values when there is presence of $30 \%$ outliers. This indicates that Jmad-statistics are not affected by the increase in sample size and still can detect a false null hypothesis when there is presence of $20 \%$ outliers. $\mathrm{Jm}$-statistics can also detect a false null hypothesis when there is presence of only $10 \%$ outliers.

Table 5. Power of test for different statistics with outliers for $n=50$

\begin{tabular}{|c|c|c|c|c|c|}
\hline & \multicolumn{5}{|c|}{$n=50$} \\
\hline outlier & $\mathbf{1 0 \%}$ & $\mathbf{2 0 \%}$ & $\mathbf{3 0 \%}$ & $\mathbf{4 0 \%}$ & $\mathbf{5 0 \%}$ \\
\hline $\begin{array}{c}\text { Jennrich } \\
\text { statistic }\end{array}$ & 1 & 1 & 1 & 1 & 1 \\
\hline Js-statistic & 0.9947 & 1 & 1 & 1 & 1 \\
\hline Jm-statistic & 0.2562 & 0.8132 & 0.992 & 1 & 1 \\
\hline Jmad-statistic & 0.0309 & 0.4428 & 0.9899 & 0.9997 & 1 \\
\hline
\end{tabular}

For large sample size $n=50$ and $n=100, J m$-statistic and Jmad-statistic are not affected to detect a false null hypothesis with the presence of $10 \%$ outliers. However, only Jmad-statistic is not affected to detect a false null hypothesis with the presence of $20 \%$ outliers when $n=50$. This analysis shows that $J m$-statistic and Jmad-statistic can overcome outlier problems into Jennrich statistic in testing of correlation matrix hypothesis. Jmad-statistic is superior in testing correlation hypothesis for different sample sizes, especially those involving $10 \%$ outliers. Therefore, a robust method can be used as an alternative in reducing the influence of outliers.

Table 6. Power of test for different statistics with outliers for $n=100$

\begin{tabular}{|c|c|c|c|c|c|}
\hline & \multicolumn{5}{|c|}{$\mathbf{N = 1 0 0}$} \\
\hline Outlier & $\mathbf{1 0 \%}$ & $\mathbf{2 0 \%}$ & $\mathbf{3 0 \%}$ & $\mathbf{4 0 \%}$ & $\mathbf{5 0 \%}$ \\
\hline Jennrich statistic & 1 & 1 & 1 & 1 & 1 \\
\hline JS-statistic & 1 & 1 & 1 & 1 & 1 \\
\hline Jm-statistic & 0.4843 & 0.9805 & 1 & 1 & 1 \\
\hline Jmad-statistic & 0.0691 & 0.8025 & 1 & 1 & 1 \\
\hline
\end{tabular}

\subsection{Real Data for Testing the Equality of Two Matrices}

The largest economic disturbance is happened after Great Recession in 2007 until 2009. It is about eighteen months recession which is the longest time period [29]. This recession gives a bad impact to job market, unemployment rate, social systems, and stocks market. Specifically, the stock market crash occurred in September 2008. The rise and collapse of correlation are important subject in inspecting the stock behaviour. It may help the investors utilize which stocks that impact correlation in order to improve asset allocation decisions [30](Mc Bride, 2011). To illustrate the application of Jmad-statistic, we used 81 stocks that have been traded at Bursa Malaysia from year 2009. All of the stocks having more than RM500 million market capital. The time series data of 81 stocks 
for two different time period is retrieved from Bloomberg database. The first time period is start from Jan until June 2009 (6 months before recession end), and the second time period is start from July 2009 - December 2009 (6 months after recession end).

Then we conduct a hypothesis testing to determine the equality of two correlation matrices, $H_{0}: P_{1}=P_{2}$ and the alternative hypothesis is $H_{1}: P_{1} \neq P_{2}$. The statistical test value from Jmad-statistical test is 11433.08. Based on Chi-square distribution with $\frac{p(p-1)}{2}=\frac{81(81-1)}{2}=3240$ degree of freedom, the critical values is 3108.74 . Therefore, there is enough evidence to reject the null hypothesis and conclude that the Bursa Malaysia also having risen and collapse during the great recession.

\section{Conclusions}

The Jennrich statistic is appropriate way to test the correlation between 2 or more independent correlation matrices. However, this statistic is extremely sensitive to the presence of outliers that can reduce the test's power. To address the presence of outliers, robust correlations, such as median correlation and median absolute deviation correlation, as well as Spearman correlation, can be used to integrate into Jennrich statistic. These proposed methods are alternative methods in reducing the influence of outliers, especially in comparing correlation hypothesis testing. Js-statistic, Jm-statistic and Jmad-statistic are proposed and have been compared to classical Jennrich statistic using the power of test values. For perfect data, which have no outliers in the data set, all 4 statistical tests for correlation analysis show that the probability value of the test of significance will detect a low deviation from the null hypothesis when the correlation difference varies up to 0.2 . In addition, $\mathrm{Jm}$-statistic and $\mathrm{Jmad}$-statistic have low probability values to detect a deviation from the null hypothesis when the correlation difference varies up to 0.4. When outliers exist in the dataset, Jmad-statistic still gives a strong performance compared to other methods. This proves that, although outliers exist in the data, the Jmad-statistic does not affect the hypothesis testing to compare correlation matrices. The classical Jennrich statistic also suffer greatly from the presence of outliers even though only $10 \%$ of outliers are present in the dataset.

\section{Acknowledgements}

This research is part of a $\mathrm{PhD}$ study from the Awang Had Salleh Graduate School at Universiti Utara Malaysia. The authors appreciate the help they received from Universiti Utara Malaysia. We also appreciate the helpful recommendations provided by the anonymous reviewers.

\section{REFERENCES}

[1] Y. Aït-sahalia and D. Xiu, "Using principal component analysis to estimate a high dimensional factor model with high-frequency data," J. Econom., vol. 201, no. 2, pp. 384399, 2017, doi: 10.1016/j.jeconom.2017.08.015.

[2] P. L. H. Yu, X. Wang, and Y. Zhu, "High dimensional covariance matrix estimation by penalizing the matrix-logarithm transformed likelihood," Comput. Stat. Data Anal., vol. 114, pp. 12-25, 2017, doi: https://doi.org/10.1016/j.csda.2017.04.004.

[3] A. E. Bilgrau, R. F. Brondum, P. S. Eriksen, K. Dybkaer, and M. Bogsted, "Estimation of a common covariance matrix for multiple classes with applications in meta- and discriminant analysis," Submitt. to Ann. Appl. Stat., no. March, 2017.

[4] J. Gao and D. B. Hitchcock, "James-Stein shrinkage to improve k-means cluster analysis,” Comput. Stat. Data Anal., vol. 54, no. 9, pp. 2113-2127, 2010, doi: https://doi.org/10.1016/j.csda.2010.03.018.

[5] W. Lan, R. Luo, C. L. Tsai, H. Wang, and Y. Yang, "Testing the diagonality of a large covariance matrix in a regression setting,” J. Bus. Econ. Stat., vol. 33, no. 1, pp. 76-86, 2014.

[6] A. Belloni, V. Chernozhukov, and K. Kato, "Valid post-selection inference in high-dimensional approximately sparse quantile regression models," J. Am. Stat. Assoc., vol. 114, no. 526, pp. 749-758, Apr. 2019, doi: 10.1080/01621459.2018.1442339.

[7] A. Gupta, B. Johnson, and D. Nagar, "Testing equality of several correlation matrices,” Rev. Colomb. Estad., vol. 36, no. 2, pp. 237-258, 2013.

[8] G. E. P. Box, "A general distribution theory for a class of likelihood criteria,” Biometrika, vol. 36, no. 3-4, pp. 317346, Dec. 1949, doi: 10.1093/biomet/36.3-4.317.

[9] S. Kullback, “On testing correlation matrices,” J. R. Stat. Soc., vol. 16, no. 1, pp. 80-85, 1967.

[10] M. A. Aitkin, W. C. Nelson, and K. H. Reinfurt, "Tests for correlation matrices,” Biometrika, vol. 55, no. 2, pp. 327334, 1968.

[11] M. A. Aitkin, "Some tests for correlation matrices," Biometrika, vol. 56, no. 2, pp. 443-446, 1969.

[12] R. I. . Jennrich, "An Asymptotic chi2 test for the equality of two correlation matrices,” J. Am. Stat. Assoc., vol. 65, no. 330, pp. 904-912, 1970.

[13] S. Sharif and T. A. M. Atiany, "Testing several correlation matrices using robust approach,” Asian J. Sci. Res., vol. 11, no. 1, pp. 84-95, 2017, doi: 10.3923/ajsr.2018.84.95.

[14] M. A. Djauhari, “On distributional behavior of Jennrich’s statistic,” Malaysian J. Math. Sci., vol. 10, no. 2, pp. 233244, 2016.

[15] T. A. M. Atiany and S. Sharif, "New statistical test for testing several correlation matrices," Glob. J. Pure Appl. Math., vol. 12, no. 5, pp. 4285-4298, 2016.

[16] K. Larntz and M. D. Perlman, "A simple test for the equality of correlation matrices,” 1985. 
[17] R. R. Wilcox, D. A. Granger, and F. Clark, "Modern robust statistical methods: Basics with illustrations using psychobiological data,” Univers. J. Psychol., vol. 1, no. 2, pp. 21-31, 2013, doi: 10.13189/ujp.

[18] N. A. Ahad, N. A. Zakaria, S. Abdullah, S. S. S. Yahaya, and N. Yusof, "Robust correlation Procedure via Sn estimator,” J. Telecommun. Electron. Comput. Eng., vol. 10, no. 1-10, pp. 115-118, 2018.

[19] C. R. Pernet, R. Wilcox, and G. A. Rousselet, "Robust correlation analyses: False positive and power validation using a new open source Matlab toolbox," Front. Psychol., vol. 3, no. Jan 2013, 2013, doi: 10.3389/fpsyg.2012.00606.

[20] Waego Hadi Nugroho, Ni Wayan Surya Wardhani , Adji Achmad Rinaldo Fernandes, Solimun, "Robust Regression Analysis Study for Data with Outliers at Some Significance Levels," Mathematics and Statistics, Vol. 8, No. 4, pp. 373 381, 2020. DOI: 10.13189/ms.2020.080401.

[21] S. S. S. Yahaya, H. Ali, and Z. Omar, "An alternative hotelling T2 control chart based on minimum vector variance (MVV),” Mod. Appl. Sci., vol. 5, no. 4, pp. 132151, 2011, doi: 10.5539/mas.v5n4p132.

[22] P. M. Yuan, K.-H., \& Bentler, "Effect of outliers on estimators and tests in covariance structure,” Br. J. Math. Stat. Psychol., vol. 54, no. 1, pp. 161-175, 2001, doi: http://dx.doi.org/10.1348/000711001159366.

[23] M. Y. Wang and C. E. Zwilling, "Multivariate computing and robust estimating for outlier and novelty in data and imaging sciences,” Adv. Bioeng., 2015, doi: 10.5772/59750.
[24] G. L. Shevlyakov and P. Smirnov, "Robust estimation of the correlation coefficient: An attempt of survey," Austrian J. Stat., vol. 40, no. 1, pp. 147-156, 2011, doi: http://dx.doi.org/10.17713/ajs.v40i1\&2.206.

[25] P. J. Huber, "Robust estimation of a location parameter," Ann. Math. Stat., vol. 35, no. 1, pp. 73-101, 1964, doi: 10.1002/9781118445112.stat08190.

[26] Bahtiar Jamili Zaini , Shamshuritawati Sharif , "The Performance of Different Correlation Coefficient under Contaminated Bivariate Data," Mathematics and Statistics, Vol. 8, No. 2A, pp. 1 - 8, 2020. DOI: 10.13189/ms.2020.081301.

[27] R. C. Mittelhammer, Mathematical statistics for economics and business. 2013.

[28] S. Yue, P. Pilon, and G. Cavadias, "Power of the Mann \pm Kendall and Spearman's rho tests for detecting monotonic trends in hydrological series,” J. Hydrol., vol. 259, pp. 254271, 2002.

[29] A. L. Kalleberg and T. M. von Wachter, "The U . S . Labor Market During and After the Great Recession : Continuities and Transformations,” Russell Sage Found. J. Soc. Sci., vol. 3, no. 3, pp. 0-19, 2017, doi: 10.7758/RSF.2017.3.3.01.

[30] E. Mc Bride, "Understanding the recent rise in correlations and how you can turn it to your advantage.," Retrieved from http://www.forbes.com/sites/riabiz/2011/03/09/understandi ng-the-recent-rise-in-correlations-and-how-you-can-turn-it -to-your-advantage. 2011,Sept. 3. 